\title{
SOLUCIÓN LOCAL Y SINGULARIDAD PARA UN SISTEMA DE KIRCHHOFF NO LINEAL
}

\author{
Teófanes Quispe Méndez
}

RESUMEN.- Estudiamos la existencia y singularidad de soluciones locales del problema mixto para un sistema de Kirchhoff no lineal.

PALABRAS CLAVE.- Solución local, Singularidad de soluciones, Método de estimaciones de Tartar, Sistema de Kirchhoff no lineal, Sistema hiperbólico no lineal.

\section{LOCAL SOLUTION AND BLOW-UP FOR A NONLINEAR KIRCHHOFF'S SYSTEM}

\begin{abstract}
We study the existence and blow-up of the local solution to the
\end{abstract} mixed problem for a nonlinear Kirchhoff's system.

KEYWORDS.- Local solution, Blow-up of solutions, Tartar's estimates method, Nonlinear Kirchhoff's system, Nonlinear hyperbolic system.

\section{INTRODUCCIÓN}

Un fenómeno natural importante es el estudio desde el contexto de la Física Relativa de la interacción de ciertas partículas elementales llamadas mesones en un campo electromágnetico. Un modelo matemático que describe la interacción de los mesones es el siguiente sistema propuesto por Segal [10]

$$
\left\{\begin{array}{lll}
u_{t t}-\Delta u+\alpha^{2} u+g^{2} v^{2} u=0 & \text { en } & \Omega \times[0, \infty[ \\
v_{t t}-\Delta v+\beta^{2} v+h^{2} u^{2} v=0 & \text { en } & \partial \Omega \times[0, \infty[
\end{array}\right.
$$

donde $\alpha$ y $\beta$ son las masas de los mesones $u$ y $v$ respectivamente, $g$ y $h$ son constantes de interacción y $\Omega$ es un conjunto abierto de $\mathbb{R}^{3}$.

\footnotetext{
1 Universidad Nacional Mayor de San Marcos. Facultad de Ciencias Matemáticas. e-mail: tquispem@unmsm.edu.pe
} 
Muchos autores estudiaron desde diversos puntos de vista el sistema (1.1) y entre ellos podemos mencionar a Jörgens [2], Makhankov [4] y Medeiros \& Perla Menzala [6].

Una generalización posterior de (1.1) es el siguiente sistema de Kirchhoff no lineal

$$
\left\{\begin{array}{l}
u_{t t}-M\left(\int_{\Omega}|\nabla u|^{2} d x\right) \Delta u+m_{1}^{2} u+(\rho+2) \delta|v|^{\rho+2}|u|^{\rho} u=0 \text { en } \Omega \times[0, \infty[ \\
u_{t t}-M\left(\int_{\Omega}|\nabla v|^{2} d x\right) \Delta v+m_{2}^{2} v+(\rho+2) \delta|u|^{\rho+2}|v|^{\rho} v=0 \text { en } \Omega \times[0, \infty[
\end{array}\right.
$$

donde $M(s)$ es una función real no negativa de clase $C^{1}$ para $s \geq 0, \Omega$ es un conjunto abierto de $\mathbb{R}^{n} ; m_{1}, m_{2}, \delta$ y $\rho$ son constantes reales.

Diversos autores investigaron el sistema (1.2) y podemos mencionar algunos de ellos. La solución global: Medeiros \& Milla Miranda [5] usando el método de la energía para $M(s) \equiv 1$ y $\delta=1$; Milla Miranda \& Medeiros [7] usando el método del pozo potencial para $M(s) \equiv 1$ y $\delta=-1$. La solución local: Quispe Méndez [9] usando el método de estimativas de Tartar para $M(s) \geq m_{0}>0 \quad$ y $\delta<0$. La singularidad de las soluciones: Carrillo Díaz [1] utilizando el método de concavidad de Levine para $M(s) \equiv 1, \rho=0$ y con energía inicial no positiva; Quispe Méndez [9] utilizando el método de concavidad de Levine para $M(s)=a+b s^{\rho+1}$ y con energía inicial no positiva y positiva.

Sea $\Omega$ un conjunto abierto y acotado de $\mathbb{R}^{n}$ con frontera bien regular $\partial \Omega$. El sistema (1.2) es un caso particular del siguiente problema mixto para el sistema de Kirchhoff no lineal

$$
\left\{\begin{array}{l}
u_{t t}-M\left(\int_{\Omega}|\nabla u|^{2} d x\right) \Delta u+m_{1}^{2} u+\alpha f(u, v)+(\rho+2) \delta|v|^{\rho+2}|u|^{\rho} u=0 \text { en } \Omega \times[0, \infty[ \\
v_{t t}-M\left(\int_{\Omega}|\nabla v|^{2} d x\right) \Delta v+m_{2}^{2} v+\beta f(u, v)+(\rho+2) \delta|u|^{\rho+2}|v|^{\rho} v=0 \text { en } \Omega \times[0, \infty[
\end{array}\right.
$$

que satisface las condiciones de frontera

$$
u(x, t)=0, v(x, t)=0 \text { en } \partial \Omega \times[0, \infty[
$$

y las condiciones iniciales

$$
\left\{\begin{array}{l}
u(x, 0)=u_{0}(x), u_{t}(x, 0)=u_{1}(x) \text { en } \Omega \\
v(x, 0)=v_{0}(x), v_{t}(x, 0)=v_{1}(x) \text { en } \Omega
\end{array}\right.
$$

donde $M(s)$ es una función real de clase $C^{1}$ definida en $\left[0, \infty\left[\operatorname{con} M(s) \geq m_{0}>0, \forall s \geq 0\right.\right.$; $f(u, v)=2(\rho+2) \lambda|\alpha u+\beta v|^{2 \rho+2}(\alpha u+\beta v) ; m_{0}, m_{1}, m_{2}, \alpha, \beta, \lambda, \delta$ y $\rho$ son constantes reales.

El problema (1.3) - (1.5) fue estudiado por Li and Tsai [3] para el caso $M(s) \equiv 1$ y $\rho=0$, obteniendo la existencia de la solución local y la singularidad en tiempo finito de las soluciones. 
El propósito del presente trabajo es discutir la existencia y la singularidad en tiempo finito de las soluciones locales del problema (1.3) - (1.5). En la prueba de existencia, utilizaremos el método de Galerkin y las estimativas de Tartar, y en la discución de la singularidad, emplearemos resultados de Li and Tsai [3] con energía inicial $E(0)<0, E(0)=0$ y $E(0)>0$.

\section{PRELIMINARES}

Sea $\Omega$ un conjunto abierto y acotado de $\mathbb{R}^{n}$ con frontera bien regular $\partial \Omega$. Denotemos el producto interno y la norma de $L^{2}(\Omega)$ y $L^{p}(\Omega)$ con $\left(.\right.$, . .) y ||$_{L^{p}(\Omega)}$, respectivamente, para $1 \leq p \leq \infty$. Además $a(.,$.$) y \|$.$\| , denotarán el producto interno y la norma de H_{0}^{1}(\Omega)$, donde $a(u, v)=\int_{\Omega} \nabla u . \nabla v d x$ es la forma de Dirichlet.

Sea $X$ un espacio de Banach, $0<T \leq \infty$ y $1 \leq p \leq \infty$. Representamos con $L^{p}(0, T ; X)$ al espacio de Banach de las funciones vectoriales $u:] 0, T\left[\rightarrow X\right.$ medibles con $\|u(t)\|_{X} \in L^{p}(0, T)$, dotados de la norma:

$$
\begin{aligned}
& \|u\|_{L^{p}(0, T ; X)}:=\left(\int_{0}^{T}\|u(t)\|_{X}^{p} d t\right)^{\frac{1}{p}}, 1 \leq p<\infty, \\
& \|u\|_{L^{\infty}(0, T ; X)}:=\sup _{0<t<T} \operatorname{ess}\|u(t)\|_{X}, p=\infty .
\end{aligned}
$$

Similarmente cuando $0<T<\infty$, representamos con $C^{0}([0, T] ; X)$ al espacio de Banach de las funciones continuas $u:[0, T] \rightarrow X$, con la norma

$$
\|u\|_{C^{0}([0, T] ; X)}:=\operatorname{Sup}_{o \leq t \leq T}\|u(t)\| .
$$

También representamos con $C_{w}^{0}([0, T] ; X)$ al espacio de las funciones débilmente continuas $u:[0, T] \rightarrow X$.

Denotamos $u^{\prime}:=u_{t}, u^{\prime \prime}:=u_{t t} \quad y \quad u(t):=u(., t)$.

\section{Hipótesis:}

Supongamos que $M(s)$ es una función real que satisface:

(H1) $M \in C^{1}\left(\left[0, \infty[)\right.\right.$ y $M(s) \geq m_{0}>0, \forall s \geq 0 m_{0}$ constante real,

(H2) $(2 \gamma+1) \widehat{M}(s) \geq s M(s), \forall s \geq 0$,

donde $\gamma$ es una constante real tal que $0<\gamma<\frac{\rho+1}{2}$ con $\rho \geq 0$, y $\hat{M}(s)=\int_{0}^{s} M(\xi) d \xi$. 
Supongamos que $m_{1}, m_{2}, \alpha, \beta, \lambda$ y $\delta$ son números reales fijo con $\lambda^{2}+\delta^{2} \neq 0$ que satisfacen:

(H3) $m_{1}^{2} \xi^{2}+m_{2}^{2} \eta^{2}+\lambda|\alpha \xi+\beta \eta|^{2 \rho+4}+\delta|\xi \eta|^{\rho+2} \leq 0, \forall \xi, \eta \in \mathbb{R}$.

Lema 2.1 (Li - Tsai [3]). Sea $\gamma>0$ y sea $b(t): \mathbb{R}^{+} \rightarrow \mathbb{R}^{+}$una función de clase C que satisface

$$
b^{\prime \prime}(t)-4(\gamma+1) b^{\prime}(t)+4(\gamma+1) b(t) \geq 0, \forall t \geq 0
$$

Si $b^{\prime}(0)>r_{2} b(0)$, entonces $b^{\prime}(t)>0, \forall t>0$, donde $r_{2}=2(\gamma+1)-2 \sqrt{(\gamma+1) \gamma}$ es la menor raíz de la ecuación cuadrática $r^{2}-4(\gamma+1) r+4(\gamma+1)=0$.

Lema 2.2. (Li-Tsai [3]). Sea $\gamma>0$. Si $J(t)$ es una función no creciente en $\left[t_{0}, \infty\left[, t_{0} \geq 0\right.\right.$ y satisface la inecuación diferencial

$$
\left[J^{\prime}(t)\right]^{2} \geq a+b[J(t)]^{2+\frac{1}{\gamma}}, \forall t \geq t_{0}
$$

donde $a>0 \quad$ y $\quad b \in \mathbb{R}$, entonces existe un número real positivo $T_{*}$ tal que $\lim _{t \rightarrow T_{*}^{-}} J(t)=0$ y una cota superior de $T_{*}$ puede ser estimada, respectivamente, en los siguientes casos:

(i) Cuando $b<0$ y $J\left(t_{0}\right)<\min \left\{1, \sqrt{\frac{a}{-b}}\right\}$,

$$
T_{*} \leq t_{0}+\frac{1}{\sqrt{-b}} \ln \left(\frac{\sqrt{\frac{a}{-b}}}{\sqrt{\frac{a}{-b}}-J\left(t_{0}\right)}\right),
$$

(ii) Cuando $b=0$,

$$
T_{*} \leq t_{0}+\frac{J\left(t_{0}\right)}{\sqrt{a}}
$$

(iii) Cuando $b>0$,

$$
T_{*} \leq t_{0}+2^{\frac{3 \gamma+1}{2 \gamma}} \frac{\gamma c}{\sqrt{a}}\left\{1-\left[1+c J\left(t_{0}\right)\right]^{-\frac{1}{2 \gamma}}\right\},
$$

donde $c=\left(\frac{a}{b}\right)^{2+\frac{1}{\gamma}}$

Lema 2.3 (Desigualdad generalizada de Gronwall [8]). Sea $f:[0, \infty[\rightarrow[0, \infty[$ continua, $g:] 0, \infty[\rightarrow] 0, \infty[$ continua y no decreciente y sea $C$ una constante positiva, tal que se satisface la desigualdad

$$
f(t) \leq C+\int_{0}^{t} g(f(s)) d s, \forall t \in[0, \infty[
$$


Entonces

$$
f(t) \leq G^{-1}\left(T_{*}\right)<\infty, \forall t \in\left[0, T_{*}\right],
$$

para cualquier número fijo $T_{*}<G(\infty)$, donde

$$
G(t)=\int_{C}^{t} \frac{d s}{g(s)}, \forall t \in[C, \infty[.
$$

Más aun, si $G(\infty)=\infty$, entonces

$$
f(t) \leq G^{-1}(t), \forall t \in[0, \infty[.
$$

Definición 2.4. Un par de funciones $\{u, v\}$ es llamada solución del problema (1.3) - (1.5) sobre $[0, T[$ si las funciones $u, v: \Omega \times[0, T[\rightarrow \mathbb{R}$ satisfacen las condiciones (1.4) - (1.5) y las igualdades

$$
\begin{array}{r}
\left(u^{\prime \prime}(t), w\right)+M\left(\|u(t)\|^{2}\right) a(u(t), w)+\left(m_{1}^{2} u(t)+\alpha f(u(t), v(t))\right. \\
\left.+(\rho+2) \delta|v(t)|^{\rho+2}|u(t)|^{\rho} u(t), w\right)=0 \\
\left(v^{\prime \prime}(t), w\right)+M\left(\|v(t)\|^{2}\right) a(v(t), w)+\left(m_{2}^{2} v(t)+\beta f(u(t), v(t))\right. \\
\left.+(\rho+2) \delta|u(t)|^{\rho+2}|v(t)|^{\rho} v(t), w\right)=0
\end{array}
$$

para todo $w \in H_{0}^{1}(\Omega)$, en el sentido de las distribuciones $D^{\prime}(0, T)$.

\section{EXISTENCIA LOCAL}

La estrategia para obtener la solución local del problema (1.3) - (1.5) es primero obtener las soluciones aproximadas del problema dado por el método de Galerkin, después obtener estimativas a priori por el método de Tartar y finalmente por argumentos de compacidad de Lions, se obtiene la existencia de soluciones. Para la unicidad de soluciones, se utiliza el método estandar de la energía.

Teorema 3.1 (Solución Local). Si $M$ verifica la hipótesis (H1). $\rho \geq 0$ para $n=1,2, \rho=0$ para $n=3, u_{0}, v_{0} \in H_{0}^{1}(\Omega) \cap H^{2}(\Omega) \quad y \quad u_{1}, v_{1} \in H_{0}^{1}(\Omega)$, entonces existe un único $T_{M}, 0<T_{M} \leq \infty$ y una única solución $\{u, v\}$ del problema (1.3) - (1.5) sobre $\left[0, T_{M}[\right.$ tal que

$$
\begin{aligned}
& u, v \in L^{\infty}\left(0, T_{M} ; H_{0}^{1}(\Omega) \cap H^{2}(\Omega)\right), \\
& u^{\prime}, v^{\prime} \in L^{\infty}\left(0, T_{M} ; H_{0}^{1}(\Omega)\right), \\
& u^{\prime \prime}, v^{\prime \prime} \in L^{\infty}\left(0, T_{M} ; L^{2}(\Omega)\right) .
\end{aligned}
$$




\section{Demostración}

Soluciones Aproximadas. Sea $\left\{w_{k}\right\}$ una base hilbertiana de $H_{0}^{1}(\Omega) \cap H^{2}(\Omega)$ formada por las funciones propias generalizadas del operador elíptico $-\Delta$, esto es, satisfacen $-\Delta w_{k}=\lambda_{k} w_{k}$ en $\Omega$, $w_{k}=0$ sobre $\partial \Omega$.

Sea $V_{m}=\left[w_{1}, w_{2}, \ldots, w_{m}\right]$ el subespacio generado por los primeros m vectores $w_{1}, w_{2}, \ldots, w_{m}$ de $\left\{w_{k}\right\}$. Sean

$$
u_{m}(t)=\sum_{j=1}^{m} g_{j m}(t) w_{j}, w_{m}(t)=\sum_{j=1}^{m} h_{j m}(t) w_{j},
$$

las soluciones aproximadas en $V_{m}$ del problema (1.3) - (1.5), donde las funciones $g_{j m}(t)$ y $h_{j m}(t), j=1,2, \ldots, m$, son determinadas del siguiente problema en ecuaciones diferenciales ordinarias, para $j=1,2, \ldots, m$

$$
\begin{gathered}
\quad\left(u_{m}^{\prime \prime}(t), w_{j}\right)+M\left(\left\|u_{m}(t)\right\|^{2}\right) a\left(u_{m}(t), w_{j}\right) . \\
+m_{1}^{2}\left(u_{m}(t), w_{j}\right)+\alpha\left(f\left(u_{m}(t), v_{m}(t)\right), w_{j}\right) \\
+(\rho+2) \delta\left(\left|v_{m}\right|^{\rho+2}\left|u_{m}(t)\right|^{\rho} u_{m}(t), w_{j}\right)=0 \\
\left(v_{m}^{\prime \prime}(t), w_{j}\right)+M\left(\left\|v_{m}(t)\right\|^{2}\right) a\left(v_{m}(t), w_{j}\right) \\
+m_{2}^{2}\left(v_{m}(t), w_{j}\right)+\beta\left(f\left(u_{m}(t), v_{m}(t)\right), w_{j}\right) \\
+(\rho+2) \delta\left(\left|u_{m}\right|^{\rho+2}\left|v_{m}(t)\right|^{\rho} v_{m}(t), w_{j}\right)=0 \\
\begin{cases}u_{m}(0) & =u_{0 m}, u_{o m} \rightarrow u_{0} \quad \text { fuerte en } H_{0}^{1}(\Omega) \cap H^{2}(\Omega) \\
v_{m}(0) & =v_{0 m}, v_{o m} \rightarrow v_{0} \quad \text { fuerte en } H_{0}^{1}(\Omega) \cap H^{2}(\Omega) \\
u_{m}^{\prime}(0) & =u_{1 m}, u_{1 m} \rightarrow u_{1} \quad \text { fuerte en } H_{0}^{1}(\Omega) \\
v_{m}^{\prime}(0) & =v_{1 m}, v_{1 m} \rightarrow v_{1} \quad \text { fuerte en } H_{0}^{1}(\Omega)\end{cases}
\end{gathered}
$$

donde

$$
\begin{aligned}
& u_{o m}=\sum_{j=1}^{m} \alpha_{0 j m} w_{j}, v_{0 m}=\sum_{j=1}^{m} \beta_{0 j m} w_{j}, \\
& u_{1 m}=\sum_{j=1}^{m} \alpha_{1 j m} w_{j}, v_{1 m}=\sum_{j=1}^{m} \beta_{1 j m} w_{j} .
\end{aligned}
$$

Por el teorema de Carathéodory, aseguramos la existencia de una solución local $\left\{u_{m}, v_{m}\right\}$ del problema aproximado (3.1) - (3.3) en el intervalo $\left[0, T_{m}[\right.$. Las siguientes estimativas a priori nos permitirá extender la solución $\left\{u_{m}, v_{m}\right\}$ a un intervalo $\left[0, T_{0}\right]$ independiente de $m$. 
Estimativas a Priori I. Multiplicando (3.1) y (3.2) por $g_{m}^{\prime}(t)$ y $h_{j m}^{\prime}(t)$, respectivamente, sumando $j$ de 1 hasta $m$, después sumando miembro a miembro las dos ecuaciones, obtenemos

$$
\begin{aligned}
\frac{d}{d t}\left[\left|u_{m}^{\prime}(t)\right|_{L^{2}(\Omega)}^{2}+\left|v_{m}^{\prime}(t)\right|_{L^{2}(\Omega)}^{2}+\hat{M}\left(\left\|u_{m}(t)\right\|^{2}\right)+\hat{M}\left(\left\|v_{m}(t)\right\|^{2}\right)\right. \\
\left.+m_{1}^{2}\left|u_{m}(t)\right|_{L^{2}(\Omega)}^{2}+m_{2}^{2}\left|v_{m}(t)\right|_{L^{2}(\Omega)}^{2}\right] \\
=-2\left[\alpha\left(f\left(u_{m}(t), v_{m}(t)\right), u_{m}^{\prime}(t)\right)+\beta\left(f\left(u_{m}(t), v_{m}(t)\right), v_{m}^{\prime}(t)\right)\right] \\
-2(\rho+2) \delta\left[\left(\left|v_{m}(t)\right|^{\rho+2}\left|u_{m}(t)\right|^{\rho} u_{m}(t), u_{m}^{\prime}(t)\right)\right. \\
\left.+\left(\left|u_{m}(t)\right|^{\rho+2}\left|v_{m}(t)\right|^{\rho} v_{m}(t), v_{m}^{\prime}(t)\right)\right] .
\end{aligned}
$$

Denotemos con $\mu_{1 m}(t)$ al segundo miembro de (3.4). Aplicando las desigualdades de CauchySchwartz, Hölder y las inmersiones de Sobolev, para alguna constante $C_{1}>0$, obtenemos

$$
\begin{aligned}
\mu_{1 m}(t) & \leq C_{1}\left[\left\|u_{m}(t)\right\|^{2 \rho+3}\left|u_{m}^{\prime}(t)\right|_{L^{2}(\Omega)}+\left\|v_{m}(t)\right\|^{2 \rho+3}\left|u_{m}^{\prime}(t)\right|_{L^{2}(\Omega)}\right. \\
& +\left\|u_{m}(t)\right\|^{2 \rho+3}\left|v_{m}^{\prime}(t)\right|_{L^{2}(\Omega)}+\left\|v_{m}(t)\right\|^{2 \rho+3}\left|v_{m}^{\prime}(t)\right|_{L^{2}(\Omega)} \\
& +\left\|v_{m}(t)\right\|^{\rho+2}\left\|u_{m}(t)\right\|^{\rho+1}\left|u_{m}^{\prime}(t)\right|_{L^{2}(\Omega)} \\
& \left.+\left\|u_{m}(t)\right\|^{\rho+2}\left\|v_{m}(t)\right\|^{\rho+1}\left|v_{m}^{\prime}(t)\right|_{L^{2}(\Omega)}\right] .
\end{aligned}
$$

En lo que sigue, se emplea el método de estimativas de Tartar. Sea la función $\varphi_{m}(t)$ definida por

$$
\begin{aligned}
\varphi_{m}(t): & =\left|u_{m}^{\prime}(t)\right|_{L^{2}(\Omega)}^{2}+\left|v_{m}^{\prime}(t)\right|_{L^{2}(\Omega)}^{2}+\hat{M}\left(\left\|u_{m}(t)\right\|^{2}\right)+\hat{M}\left(\left\|v_{m}(t)\right\|^{2}\right) \\
& +m_{1}^{2}\left|u_{m}(t)\right|_{L^{2}(\Omega)}^{2}+m_{2}^{2}\left|v_{m}(t)\right|_{L^{2}(\Omega)}^{2} .
\end{aligned}
$$

Como $m_{0} s \leq \hat{M}(s), \forall s \geq 0$, luego de (3.6), obtenemos

$$
\left\{\begin{array}{l}
\left|u_{m}^{\prime}(t)\right|_{L^{2}(\Omega)} \leq \varphi_{m}^{1 / 2}(t), \quad\left|v_{m}^{\prime}(t)\right|_{L^{2}(\Omega)} \leq \varphi_{m}^{1 / 2}(t) \\
\left\|u_{m}(t)\right\| \leq \frac{1}{\sqrt{m_{0}}} \varphi_{m}^{1 / 2}(t), \quad\left\|v_{m}(t)\right\| \leq \frac{1}{\sqrt{m_{0}}} \varphi_{m}^{1 / 2}(t) .
\end{array}\right.
$$

Por (3.5) - (3.7), de (3.4) para alguna constante $C_{2}>0$, se obtiene

$$
\varphi_{m}^{\prime}(t) \leq C_{2} \varphi_{m}^{\rho+2}(t) .
$$


De (3.8) y de la desigualdad generalizada de Gronwall, existe $T_{1}>0$ y una constante $K_{1}>0$, que depende ambas de los datos del problema, tal que

$$
\left|u_{m}^{\prime}(t)\right|_{L^{2}(\Omega)}^{2}+\left|v_{m}^{\prime}(t)\right|_{L^{2}(\Omega)}^{2}+\left\|u_{m}(t)\right\|^{2}+\left\|v_{m}(t)\right\|^{2} \leq K_{1}, \forall t \in\left[0, T_{1}\right]
$$

Estimativa a Priori II. Multiplicamos (3.1) y (3.2) por $g_{j m}^{\prime}(t) \lambda_{j}$ y $h_{j m}^{\prime}(t) \lambda_{j}$, respectivamente, sumando $j$ de 1 hasta $m$, luego sumando miembro a miembro, se obtiene

$$
\begin{aligned}
& \frac{d}{d t}\left[\left\|u_{m}^{\prime}(t)\right\|^{2}+\left\|v_{m}^{\prime}(t)\right\|^{2}+M\left(\left\|u_{m}(t)\right\|^{2}\right)\left|\Delta u_{m}(t)\right|_{L^{2}(\Omega)}^{2}\right. \\
& \left.+M\left(\left\|v_{m}(t)\right\|^{2}\right)\left|\Delta v_{m}(t)\right|_{L^{2}(\Omega)}^{2}+m_{1}^{2}\left\|u_{m}(t)\right\|^{2}+m_{2}^{2}\left\|v_{m}(t)\right\|^{2}\right] \\
& \quad=-2\left[\alpha\left(\nabla f\left(u_{m}(t), v_{m}(t)\right), \nabla v_{m}^{\prime}(t)\right)\right. \\
& \left.\quad+\beta\left(\nabla f\left(u_{m}(t), v_{m}(t)\right), \nabla v_{m}^{\prime}(t)\right)\right] \\
& \quad=-2(\rho+2) \delta\left[\left(\nabla \mid\left(\left.v_{m}(t)\right|^{\rho+2}\left|u_{m}(t)\right|^{\rho} u_{m}(t)\right), \nabla u_{m}^{\prime}(t)\right)\right. \\
& \left.\quad+\left(\nabla \mid\left(\left.u_{m}(t)\right|^{\rho+2}\left|v_{m}(t)\right|^{\rho} v_{m}(t)\right), \nabla v_{m}^{\prime}(t)\right)\right] \cdot \\
& \quad+2\left[M^{\prime}\left(\left\|u_{m}(t)\right\|^{2}\right) a\left(u_{m}(t), u_{m}^{\prime}(t)\right)\left|\Delta u_{m}(t)\right|_{L^{2}(\Omega)}^{2}\right. \\
& \left.\quad+M^{\prime}\left(\left\|v_{m}(t)\right\|^{2}\right) a\left(v_{m}(t), v_{m}^{\prime}(t)\right)\left|\Delta v_{m}(t)\right|_{L^{2}(\Omega)}^{2}\right],
\end{aligned}
$$

donde $M^{\prime}(s)$ es la derivada ordinaria de $M(s)$. Denotando con $\mu_{2 m}(t)$ al segundo miembro de (3.10), y luego aplicando las desigualdades de Cauchy-Schwartz, triangular, las inmersiones de Sobolev y (3.9), para alguna constante $C_{3}>0$, se tiene

$$
\begin{aligned}
\mu_{2 m}(t) \leq & c_{3}\left[\left|\Delta u_{m}(t)\right|_{L^{2}(\Omega)}^{2 \rho+2}\left\|u_{m}^{\prime}(t)\right\|+\left|\Delta v_{m}(t)\right|_{L^{2}(\Omega)}^{\rho \rho+2}\left\|u_{m}^{\prime}(t)\right\|\right. \\
& +\left|\Delta u_{m}(t)\right|_{L^{2}(\Omega)}^{\rho \rho+2}\left\|v_{m}^{\prime}(t)\right\|+\left|\Delta v_{m}(t)\right|_{L^{2}(\Omega)}^{2 \rho+2}\left\|v_{m}^{\prime}(t)\right\| \\
& +\left|\Delta u_{m}(t)\right|_{L^{2}(\Omega)}^{\rho+1}\left|\Delta v_{m}(t)\right|_{L^{2}(\Omega)}^{\rho+1}\left\|u_{m}^{\prime}(t)\right\| \\
& +\left|\Delta v_{m}(t)\right|_{L^{2}(\Omega)}^{\rho+2}\left|\Delta u_{m}(t)\right|_{L^{2}(\Omega)}^{\rho}\left\|u_{m}^{\prime}(t)\right\| \\
& +\left|\Delta v_{m}(t)\right|_{L^{2}(\Omega)}^{\rho+1}\left|\Delta u_{m}(t)\right|_{L^{2}(\Omega)}^{\rho+1}\left\|v_{m}^{\prime}(t)\right\| \\
& +\left|\Delta u_{m}(t)\right|_{L^{2}(\Omega)}^{\rho+2}\left|\Delta v_{m}(t)\right|_{L^{2}(\Omega)}^{\rho}\left\|v_{m}^{\prime}(t)\right\| \\
& \left.+\left|\Delta u_{m}(t)\right|_{L^{2}(\Omega)}^{2}\left\|u_{m}^{\prime}(t)\right\|+\left|\Delta v_{m}(t)\right|_{L^{2}(\Omega)}^{2}\left\|v_{m}^{\prime}(t)\right\|\right]
\end{aligned}
$$

A continuación, nuevamente emplearemos el método de estimativas de Tartar. Sea la función $\psi_{m}(t)$ definida por 


$$
\begin{aligned}
\psi_{m}(t): & =\left\|u_{m}^{\prime}(t)\right\|^{2}+\left\|v_{m}^{\prime}(t)\right\|^{2}+M\left(\left\|u_{m}(t)\right\|^{2}\right)\left|\Delta u_{m}(t)\right|_{L^{2}(\Omega)}^{2} \\
& +M\left(\left\|v_{m}(t)\right\|^{2}\right)\left|\Delta v_{m}(t)\right|_{L^{2}(\Omega)}^{2}+m_{1}^{2}\left\|u_{m}(t)\right\|^{2}+m_{2}^{2}\left\|v_{m}(t)\right\|^{2}
\end{aligned}
$$

Como $0<m_{0} \leq M(s), \forall s \geq 0$, de (3.12), se obtiene

$$
\left\{\begin{array}{l}
\left\|u_{m}^{\prime}(t)\right\| \leq \psi_{m}^{1 / 2}(t),\left\|v_{m}^{\prime}(t)\right\| \leq \psi_{m}^{1 / 2}(t), \\
\left|\Delta u_{m}\right|_{L^{2}(\Omega)} \leq \frac{1}{\sqrt{m_{0}}} \psi_{m}^{1 / 2}(t),\left|\Delta v_{m}(t)\right|_{L^{2}(\Omega)} \leq \frac{1}{\sqrt{m_{0}}} \psi_{m}^{1 / 2}(t) .
\end{array}\right.
$$

De (3.10), por (3.11) - (3.13), para alguna constante $C_{4}>0$, se tiene

$$
\psi_{m}^{\prime}(t) \leq C_{4}\left[\psi_{m}^{\rho+3 / 2}(t)+\psi_{m}^{3 / 2}\right] .
$$

De (3.14) y por la desigualdad generalizada de Gronwall, existe $T_{2}>0$ y una constante $K_{2}>0$, que dependen ambas de los datos iniciales del problema, tal que

$$
\left\|u_{m}^{\prime}(t)\right\|^{2}+\left\|v_{m}^{\prime}(t)\right\|^{2}+\left|\Delta u_{m}(t)\right|_{L^{2}(\Omega)}^{2}+\left|\Delta v_{m}(t)\right|_{L^{2}(\Omega)}^{2} \leq K_{2}, \forall t \in\left[0, T_{2}\right] .
$$

Estimativa a Priori III. Multiplicando (3.1) y (3.2) por $g_{j m}^{\prime \prime}(t)$ y $h_{j m}^{\prime \prime}(t)$, respectivamente, sumando $j$ de 1 hasta $m$, resultan

$$
\begin{aligned}
\left|u_{m}^{\prime \prime}(t)\right|_{L^{2}(\Omega)}^{2}= & M\left(\left\|u_{m}(t)\right\|^{2}\right)\left(\Delta u_{m}(t), u_{m}^{\prime \prime}(t)\right)-m_{1}^{2}\left(u_{m}(t), u_{m}^{\prime \prime}(t)\right) \\
& -\alpha\left(f\left(u_{m}(t), v_{m}(t)\right), u_{m}^{\prime \prime}(t)\right) \\
& -(\rho+2) \delta\left(\left|v_{m}(t)\right|^{\rho+2}\left|u_{m}(t)\right|^{\rho} u_{m}(t), u_{m}^{\prime \prime}(t)\right), \\
\left|v_{m}^{\prime \prime}(t)\right|_{L^{2}(\Omega)}^{2}= & M\left(\left\|v_{m}(t)\right\|^{2}\right)\left(\Delta v_{m}(t), v_{m}^{\prime \prime}(t)\right)-m_{2}^{2}\left(v_{m}(t), v_{m}^{\prime \prime}(t)\right) \\
& -\beta\left(f\left(u_{m}(t), v_{m}(t)\right), v_{m}^{\prime \prime}(t)\right) \\
& -(\rho+2) \delta\left(\left|u_{m}(t)\right|^{\rho+2}\left|v_{m}(t)\right|^{\rho} v_{m}(t), v_{m}^{\prime \prime}(t)\right)
\end{aligned}
$$

Por desigualdad de Cauchy - Schwartz y simplificando, se obtienen

$$
\begin{aligned}
\left|u_{m}^{\prime \prime}(t)\right|_{L^{2}(\Omega)} \leq & M\left(\left\|u_{m}(t)\right\|^{2}\right)\left|\Delta u_{m}(t)\right|_{L^{2}(\Omega)}+m_{1}^{2}\left|u_{m}(t)\right|_{L^{2}(\Omega)} \\
& -|\alpha|\left|f\left(u_{m}(t), v_{m}(t)\right)\right|_{L^{2}(\Omega)} \\
& +\left.\left.(\rho+2)|\delta||| v_{m}(t)\right|^{\rho+2}\left|u_{m}(t)\right|^{\rho} u_{m}(t)\right|_{L^{2}(\Omega)} \\
\left|v_{m}^{\prime \prime}(t)\right|_{L^{2}(\Omega)} \leq & M\left(\left\|v_{m}(t)\right\|^{2}\right)\left|\Delta v_{m}(t)\right|_{L^{2}(\Omega)}+m_{2}^{2}\left|v_{m}(t)\right|_{L^{2}(\Omega)} \\
& +\left|\beta \| f\left(u_{m}(t), v_{m}(t)\right)\right|_{L^{2}(\Omega)} \\
& +\left.\left.(\rho+2)|\delta||| u_{m}(t)\right|^{\rho+2}\left|v_{m}(t)\right|^{\rho} v_{m}(t)\right|_{L^{2}(\Omega)}
\end{aligned}
$$


Por la desigualdad de Hölder, las inmersiones de Sobolev, utilizando (3.9) y (3.15), y tomando $T_{0}=\min \left\{T_{1}, T_{2}\right\}$, existe una constante $K_{3}>0$ tal que

$$
\left|u_{m}^{\prime \prime}(t)\right|_{L^{2}(\Omega)}^{2}+\left|v_{m}^{\prime \prime}(t)\right|_{L^{2}(\Omega)}^{2} \leq K_{3}, \forall t \in\left[0, T_{0}\right]
$$

Solución sobre $\left[0, T_{0}\right]$. Con las estimativas (3.9), (3.15) y (3.16) y con argumentos de compacidad de Lions, se deduce que existe un par de funciones $\{u, v\}$ que verifica el problema (1.3) - (1.5) sobre $\left[0, T_{0}\right]$ tal que

$$
\left\{\begin{array}{l}
u, v \in L^{\infty}\left(0, T_{0} ; H_{0}^{1}(\Omega) \cap H^{2}(\Omega)\right) \\
u^{\prime}, v^{\prime} \in L^{\infty}\left(0, T_{0} ; H_{0}^{1}(\Omega)\right) \\
u^{\prime \prime}, v^{\prime \prime} \in L^{\infty}\left(0, T_{0} ; L^{2}(\Omega)\right)
\end{array}\right.
$$

Procediendo como en Quispe Méndez [9], utilizando el método estándar de la energía, se obtiene la unicidad de soluciones sobre $\left[0, T_{0}\right]$.

Solución sobre $\left[0, T_{M}\left[\right.\right.$. Determinemos el intervalo maximal $\left[0, T_{M}[\right.$ de la solución del problema (1.3) - (1.5). Desde que $\{u, v\}$ es solución del problema (3) - (5) sobre $\left[0, T_{0}\right]$, se obtiene

$$
\frac{d}{d t} E(t)=0,0 \leq t \leq T_{0}
$$

donde

$$
\begin{aligned}
E(t): & \frac{1}{2}\left[\left|u^{\prime}(t)\right|_{L^{2}(\Omega)}^{2}+\left|v^{\prime}(t)\right|_{L^{2}(\Omega)}^{2}+\hat{M}\left(\|u(t)\|^{2}\right)+\left(\|v(t)\|^{2}\right)\right. \\
& +m_{1}^{2}|u(t)|_{L^{2}(\Omega)}^{2}+m_{2}^{2}|v(t)|_{L^{2}(\Omega)}^{2}+2 \lambda|\alpha u(t)+\beta v(t)|_{L^{2 \rho+4}(\Omega)}^{2 \rho+4} \\
& \left.+2 \delta|u(t) v(t)|_{L^{\rho+2}(\Omega)}^{\rho+2}\right] .
\end{aligned}
$$

Por (3.17) y (3.18), resulta que $E$ es una función continua en $\left[0, T_{0}\right]$. También de (3.17), se obtiene

$$
\left\{\begin{array}{l}
u, v \in C_{w}^{0}\left(\left[0, T_{0}\right] ; H_{0}^{1}(\Omega) \cap H^{2}(\Omega)\right) \\
u^{\prime}, v^{\prime} \in C_{w}^{0}\left(\left[0, T_{0}\right] ; H_{0}^{1}(\Omega)\right) .
\end{array}\right.
$$

Desde que E es continua en $\left[0, T_{0}\right]$ y (3.19), se deduce 


$$
\left\{\begin{array}{l}
u, v \in C^{0}\left(\left[0, T_{0}\right] ; H_{0}^{1}(\Omega) \cap H^{2}(\Omega)\right) \\
u^{\prime}, v^{\prime} \in C^{0}\left(\left[0, T_{0}\right] ; H_{0}^{1}(\Omega)\right) .
\end{array}\right.
$$

De (3.20), existen $u\left(T_{0}\right), v\left(t_{0}\right) \in H_{0}^{1}(\Omega) \cap H^{2}(\Omega)$ y $u^{\prime}\left(T_{0}\right), v^{\prime}\left(T_{0}\right) \in H_{0}^{1}(\Omega)$.

Por los mismos argumentos para la solución $\{u, v\}$ sobre $\left[0, T_{0}\right]$, existe $T_{1}>0$ y una única solución $\left\{u^{1}, v^{1}\right\}$ del problema (1.3) - (1.5) sobre $\left[0, T_{1}\right]$ con los datos iniciales $u\left(T_{0}\right), v\left(T_{0}\right), u^{\prime}\left(T_{0}\right), v^{\prime}\left(T_{0}\right)$.

Definamos las funciones

$$
\begin{aligned}
& w^{1}(t)= \begin{cases}u(t), & \text { si } 0 \leq t \leq T_{0} \\
u^{1}\left(t-T_{0}\right), & \text { si } T_{0} \leq t \leq T_{0}+T_{1},\end{cases} \\
& w^{2}(t)=\left\{\begin{array}{lll}
v(t), & \text { si } 0 \leq t \leq T_{0} \\
v^{1}\left(t-T_{0}\right), & \text { si } T_{0} \leq t \leq T_{0}+T_{1} .
\end{array}\right.
\end{aligned}
$$

Entonces $\left\{w^{1}, w^{2}\right\}$ es una única solución del problema (1.3) - (1.5) sobre $\left[0, T_{0}+T_{1}\right]$ con los datos iniciales $u_{0}, v_{0} \in H_{0}^{1}(\Omega) \cap H^{2}(\Omega)$ y $u_{1}, v_{1} \in H_{0}^{1}(\Omega)$.

Ahora consideremos la familia $\left\{u^{i}, v^{i}\right\}_{i \in I}$ de todas las soluciones del problema (1.3) - (1.5) sobre $\left[0, T_{i}\right]$ con los datos iniciales $u_{0}, v_{0} \in H_{0}^{1}(\Omega) \cap H^{2}(\Omega)$ y $u_{1}, v_{1} \in H_{0}^{1}(\Omega)$. Si $T_{i}<T_{j}$, la unicidad de soluciones implica que $\left\{u^{i}(t), v^{i}(t)\right\}$ y $\left\{u^{j}(t), v^{j}(t)\right\}$ coinciden en $\left[0, T_{i}\right]$. Entonces el intervalo $\left[0, T_{M}[\right.$ dado por

$$
\left[0, T_{M}\left[=\bigcup_{i \in I}\left[0, T_{i}\right]\right.\right.
$$

es el intervalo máximal de existencia de la solución $\{u, v\}$ del problema (1.3) - (1.5). Esto concluye la demostración del Teorema.

\section{SINGULARIDAD DE SOLUCIONES}

El objetivo de esta sección es discutir la singularidad en tiempo finito de las soluciones del problema (1.3) - (1.5) sobre un intervalo maximal $\left[0, T_{M}[\right.$, es decir que una solución $\{u, v\}$ del problema $(1.3)-(1.5)$ sobre $\left[0, T_{M}[\right.$ tiene la propiedad 


$$
T_{M}<\infty y \lim _{\mathrm{t} \rightarrow \mathrm{T}_{\mathrm{M}}}\left[|u(t)|_{L^{2}(\Omega)}^{2}+|v(t)|_{L^{2}(\Omega)}^{2}\right]=\infty .
$$

Definición 4.1. La función energía $E(t)$ del problema (1.3) - (1.5) sobre $\left[0, T_{M}[\right.$ se define por

$$
\begin{aligned}
E(t):= & \frac{1}{2}\left[\left|u^{\prime}(t)\right|_{L^{2}(\Omega)}^{2}+\left|v^{\prime}(t)\right|_{L^{2}(\Omega)}^{2}+\widehat{M}\left(\|u(t)\|^{2}\right)+\widehat{M}\left(\|v(t)\|^{2}\right)\right. \\
& +m_{1}^{2}|u(t)|_{L^{2}(\Omega)}^{2}+m_{2}^{2}|v(t)|_{L^{2}(\Omega)}^{2}+2 \lambda|\alpha u(t)+\beta v(t)|_{L^{2 \rho+4}(\Omega)}^{2 \rho+4} \\
& \left.+2 \delta|u(t) v(t)|_{L^{\rho+2}(\Omega)}^{\rho+2}\right], 0 \leq t<T_{M},
\end{aligned}
$$

donde $\widehat{M}(s):=\int_{0}^{s} M(\xi) d \xi$.

Lema 4.2. Si $\{u, v\}$ es una solución del problema (1.3) - (1.5) sobre $\left[0, T_{M}[\right.$, entonces

$$
E(t)=E(0), 0 \leq t<T_{M}, \quad
$$

donde $E(0)$ es la energía inicial definida por

$$
\begin{aligned}
E(0): & =\frac{1}{2}\left[\left|u_{1}\right|_{L^{2}(\Omega)}^{2}+\left|v_{1}\right|_{L^{2}(\Omega)}^{2}+\widehat{M}\left(\left\|u_{0}\right\|^{2}\right)+\widehat{M}\left(\left\|v_{0}\right\|^{2}\right)\right. \\
& +m_{1}^{2}\left|u_{0}\right|_{L^{2}(\Omega)}^{2}+m_{2}^{2}\left|v_{0}\right|_{L^{2}(\Omega)}^{2}+2 \lambda\left|\alpha u_{0}+\beta v_{0}\right|_{L^{2 \rho+4}(\Omega)}^{2 \rho+4} \\
& \left.+2 \delta\left|u_{0} v_{0}\right|_{L^{\rho+2}(\Omega)}^{\rho+2}\right] .
\end{aligned}
$$

Demostración. Multiplicando las ecuaciones (1.3) por $u_{t}$ y $v_{t}$, respectivamente, integrando sobre $\Omega$, aplicando el teorema de la divergencia y sumando miembro a miembro, obtenemos $E^{\prime}(t)=0$. De aquí se obtiene el resultado.

Lema 4.3. Si $\{u, v\}$ es una solución del problema (1.3) - (1.5) sobre $\left[0, T_{M}[\right.$, entonces

$$
\begin{aligned}
2\left[\left|u^{\prime}(t)\right|_{L^{2}(\Omega)}^{2}+\left|v^{\prime}(t)\right|_{L^{2}(\Omega)}^{2}\right]= & \frac{1}{2} A^{\prime \prime}(t)+2 E(0) \\
& +2(\rho+1) \lambda|\alpha u(t)+\beta v(t)|_{L^{2 \rho+4}(\Omega)}^{2 \rho+4} \\
& +2(\rho+1) \delta|u(t) v(t)|_{L^{2 \rho+4}(\Omega)}^{2 \rho+4} \\
& +M\left(\|u(t)\|^{2}\right)\|u(t)\|^{2}-\hat{M}\left(\|u(t)\|^{2}\right) \\
& +M\left(\|v(t)\|^{2}\right)\|v(t)\|^{2}-\hat{M}\left(\|v(t)\|^{2}\right), 0 \leq t<T_{M},
\end{aligned}
$$




$$
\begin{aligned}
M & \left(\|u(t)\|^{2}\right)\|u(t)\|^{2}+\hat{M}\left(\|u(t)\|^{2}\right) \\
+ & M\left(\|v(t)\|^{2}\right)\|v(t)\|^{2}+\hat{M}\left(\|v(t)\|^{2}\right) \\
= & 2 E(0)-\frac{1}{2} A^{\prime \prime}(t)-\left[2 m_{1}^{2}|u(t)|_{L^{2}(\Omega)}^{2}+2 m_{2}^{2}|v(t)|_{L^{2}(\Omega)}^{2}\right. \\
& +2(\rho+3) \lambda|\alpha u(t)+\beta v(t)|_{L^{2 \rho+4}(\Omega)}^{2 \rho+4}, \\
& \left.+2(\rho+3) \delta|u(t) v(t)|_{L^{L^{+2}(\Omega)}}^{\rho+2}\right], \quad 0 \leq t<T_{M},
\end{aligned}
$$

donde

$$
A(t):=|u(t)|_{L^{2}(\Omega)}^{2}+|v(t)|_{L^{2}(\Omega)}^{2} .
$$

Demostración. Derivando (4.4) una y dos veces, obtenemos

$$
A^{\prime}(t)=2\left(u(t), u^{\prime}(t)\right)+2\left(v(t), v^{\prime}(t)\right)
$$

y

$$
\begin{aligned}
A^{\prime \prime}(t)= & 2\left[\left|u^{\prime}(t)\right|_{L^{2}(\Omega)}^{2}+\left|v^{\prime}(t)\right|_{L^{2}(\Omega)}^{2}\right]-2\left[M\left(\|u(t)\|^{2}\right)\|u(t)\|^{2}\right. \\
& +M\left(\|v(t)\|^{2}\right)\|v(t)\|^{2}+m_{1}^{2}|u(t)|_{L^{2}(\Omega)}^{2}+m_{2}^{2}|v(t)|_{L^{2}(\Omega)}^{2} \\
& +2(\rho+2) \lambda|\alpha u(t)+\beta v(t)|_{L^{2 \rho+4}(\Omega)}^{2 \rho+4} \\
& \left.+2(\rho+2) \delta|u(t) v(t)|_{L^{\rho+2}(\Omega)}^{\rho+2}\right] .
\end{aligned}
$$

Por (4.1) y (4.6) resultan, respectivamente,

$$
\begin{aligned}
\hat{M}\left(\|u(t)\|^{2}\right)+\hat{M}\left(\|v(t)\|^{2}\right) & =2 E(0)-\left[\left|u^{\prime}(t)\right|_{L^{2}(\Omega)}^{2}+\left|v^{\prime}(t)\right|_{L^{2}(\Omega)}^{2}\right. \\
& +m_{1}^{2}|u(t)|_{L^{2}(\Omega)}^{2}+m_{2}^{2}|v(t)|_{L^{2}(\Omega)}^{2} \\
& +2 \lambda|\alpha u(t)+\beta v(t)|_{L^{\rho \rho+4}(\Omega)}^{2 \rho+4} \\
& \left.+2 \delta|u(t) v(t)|_{L^{\rho+2}(\Omega)}^{\rho+2}\right]
\end{aligned}
$$

y

$$
\begin{aligned}
M\left(\|u(t)\|^{2}\right)\|u(t)\|^{2}+M\left(\|v(t)\|^{2}\right) \| & v(t) \|^{2} \\
= & \left|u^{\prime}(t)\right|_{L^{2}(\Omega)}^{2}+\left|v^{\prime}(t)\right|_{L^{2}(\Omega)}^{2}-\frac{1}{2} A^{\prime \prime}(t) \\
& -\left[m_{1}^{2}|u(t)|_{L^{2}(\Omega)}^{2}+m_{2}^{2}|v(t)|_{L^{2}(\Omega)}^{2}\right. \\
& +2(\rho+2) \lambda|\alpha u(t)+\beta v(t)|_{L^{2 \rho+4}(\Omega)}^{2 \rho+4} \\
& \left.+2(\rho+2) \delta|u(t) v(t)|_{L^{\rho+2}(\Omega)}^{\rho+2}\right] .
\end{aligned}
$$


Sumando estos dos resultados, se obtiene (4.3).

Lema 4.4. Supongamos que se verifican las hipótesis (H2) - (H3), sea $\{u, v\}$ una solución del problema (1.7) - (1.5) sobre $\left[0, T_{M}[y\right.$ uno de los siguientes casos se satisface:

(i) $E(0)<0$,

(ii) $E(0)=0 \quad y \quad A^{\prime}(0)=0$,

(iii) $E(0)>0 y A^{\prime}(0)>[2(\gamma+1)-2 \sqrt{(\gamma+1) \gamma}]\left[A(0)+\frac{(1+2 \gamma) E(0)}{1+\gamma}\right]$.

Entonces $\left.A^{\prime}(t)>0, \forall t \in\right] t_{0}, T_{M}\left[\right.$, donde $t_{0}:=\operatorname{máx}\left\{\frac{A^{\prime}(0)}{4(1+2 \gamma) E(0)}, 0\right\}$ en el caso (i) y $t_{0}:=0$ en los casos (ii) y (iii).

Demostración. Sea la función $J(t)$ definida por

$$
J(t):=[A(t)]^{-\gamma}, \forall t \in\left[0, T_{M}[\right.
$$

Derivando (4.7) una y dos veces, obtenemos

$$
J^{\prime}(t)=-\gamma[A(t)]^{-(\gamma+1)} A^{\prime}(t)
$$

y

$$
J^{\prime \prime}(t)=\gamma[A(t)]^{-(\gamma+2)}\left[(\gamma+1)\left[A^{\prime}(t)\right]^{2}-A(t) A^{\prime \prime}(t)\right]
$$

De (4.5) por la desigualdad de Cauchy - Schwarz y la desigualdad triangular, se obtiene

$$
\left|A^{\prime}(t)\right|^{2} \leq 4 A(t)\left[\left|u^{\prime}(t)\right|_{L^{2}(\Omega)}^{2}+\left|v^{\prime}(t)\right|_{L^{2}(\Omega)}^{2}\right]
$$

De (4.9) y (4.10), se tiene

$$
J^{\prime \prime}(t) \leq-\gamma[A(t)]^{-(\gamma+1)} K(t),
$$

donde

$$
K(t):=A^{\prime \prime}(t)-4(\gamma+1)\left[\left|u^{\prime}(t)\right|_{L^{2}(\Omega)}^{2}+\left|v^{\prime}(t)\right|_{L^{2}(\Omega)}^{2}\right]
$$

Por (4.2) y (4.3), obtenemos 


$$
\begin{aligned}
K(t)= & -4(2 \gamma+1) E(0)+2\left[(2 \gamma+1) \hat{M}\left(\|u(t)\|^{2}\right)\right. \\
& -M\left(\|u(t)\|^{2}\right)\|u(t)\|^{2} \\
& \left.+(2 \gamma+1) \hat{M}\left(\|v(t)\|^{2}\right)-M\left(\|v(t)\|^{2}\right)\|v(t)\|^{2}\right] \\
& +4(2 \gamma-\rho-1)\left[m_{1}^{2}|u(t)|_{L^{2}(\Omega)}^{2}+m_{2}^{2}|v(t)|_{L^{2}(\Omega)}^{2}\right. \\
& \left.\lambda|\alpha u(t)+\beta v(t)|_{L^{2 \rho+4}(\Omega)}^{2 \rho+4}+\delta|u(t) v(t)|_{L^{\rho+2}(\Omega)}^{\rho+2}\right] \\
& +4(\rho+1-\gamma)\left[m_{1}^{2}|u(t)|_{L^{2}(\Omega)}^{2}+m_{2}^{2}|v(t)|_{L^{2}(\Omega)}^{2}\right] .
\end{aligned}
$$

Por las hipótesis (H2) y (H3), de (4.13), resulta

$$
A^{\prime \prime}(t)-4(\gamma+1)\left[\left|u^{\prime}(t)\right|_{L^{2}(\Omega)}^{2}+\left|v^{\prime}(t)\right|_{L^{2}(\Omega)}^{2}\right] \geq-4(1+2 \gamma) E(0) .
$$

Ahora vamos a considerar tres casos diferentes de acuerdo al signo de la energía inicial $E(0)$.

(i) $\operatorname{Si} E(0)<0$, por integración de (4.14), resulta

$$
A^{\prime}(t) \geq A^{\prime}(0)-4(2 \gamma+1) E(0) t, \text { para } t \geq 0 \text {. }
$$

Así tenemos $A^{\prime}(t)>0$, para $t>t_{0}$, donde

$$
t_{0}:=\operatorname{máx}\left\{\frac{A^{\prime}(0)}{4(2 \gamma+1) E(0)}, 0\right\} \text {. }
$$

(ii) Si $E(0)=0$ y $A^{\prime}(0)>0$, entonces $A^{\prime \prime}(t) \geq 0$, para $t \geq 0$. Integrando resulta $A^{\prime}(t)>0$ para $t>0$.

(iii) Si $E(0)>0$ y $A^{\prime}(0)>r_{2}\left[A(0)+\frac{(2 \gamma+1) E(0)}{\gamma+1}\right]$, donde $r_{2}:=2(\gamma+1)-2 \sqrt{(\gamma+1) \gamma}$. De (4.5), por la desigualdad triangular, resulta

$$
A^{\prime}(t) \leq A(t)+\left|u^{\prime}(t)\right|_{L^{2}(\Omega)}^{2}+\left|v^{\prime}(t)\right|_{L^{2}(\Omega)}^{2}
$$

De (4.14) y (4.15) se tiene

$$
A^{\prime \prime}(t)-4(\gamma+1) A^{\prime}(t)+4(\gamma+1) A(t)+4(1+2 \gamma) E(0) \geq 0
$$

Definamos la función 


$$
b(t):=A(t)+\frac{(1+2 \gamma) E(0)}{1+\gamma}, \text { para } t \geq 0
$$

Entonces $b(t)$ satisface las condiciones del Lema 2.1. Asi se tiene $A^{\prime}(t)>0$, para $t>0$.

Teorema 4.5 (Singularidad de Soluciones). Supongamos que se verifican las hipótesis (H1) (H3). si $\{u, v\}$ es una solución del problema (3) - (5) sobre $\left[0, T_{M}[\right.$ con datos iniciales $u_{0}, v_{0} \in H_{0}^{1}(\Omega) \cap H^{2}(\Omega)$ y $u_{1}, v_{1} \in H_{0}^{1}(\Omega)$ satisfaciendo uno de los siguientes casos:

(i) $E(0)<0$,

(ii) $E(0)=0 \quad y \quad A^{\prime}(0)>0$,

(iii) $\frac{\left[A^{\prime}(0)\right]^{2}}{8 A(0)}>E(0)$ y $A^{\prime}(0)>r_{2}\left[A(0)+\frac{(1+2 \gamma) E(0)}{1+\gamma}\right]$,

entonces $T_{M}<\infty$ y $\operatorname{lím}_{t \rightarrow T_{M}^{-}} A(t)=\infty$. Además el tiempo finito $T_{M}$ es estimado, en el caso (i),

$$
T_{M} \leq t_{0}-\frac{J\left(t_{0}\right)}{J^{\prime}\left(t_{0}\right)}
$$

Además, si $J\left(t_{0}\right) \leq \operatorname{mín}\left\{1, \sqrt{\frac{a}{-b}}\right\}$, entonces

$$
T_{M} \leq t_{0}+\frac{1}{\sqrt{-b}} \ln \left(\frac{\sqrt{\frac{a}{-b}}}{\sqrt{\frac{a}{-b}}-J\left(t_{0}\right)}\right)
$$

En el caso (ii),

$$
T_{M} \leq t_{0}+\frac{J\left(t_{0}\right)}{J^{\prime}\left(t_{0}\right)}
$$

En el caso (iii)

$$
T_{M} \leq t_{0}+2^{\frac{3 \gamma+1}{2 \gamma}} \frac{\gamma c}{\sqrt{a}}\left[1-\left[1+c J\left(t_{0}\right)\right]^{-\frac{1}{2 \gamma}}\right] .
$$

Aqui $c:=\left(\frac{a}{b}\right)^{2+\frac{1}{\gamma}}$ con $a:=\gamma^{2}\left[A\left(t_{0}\right)\right]^{-2(\gamma+1)}\left\{\left[A^{\prime}\left(t_{0}\right)\right]^{2}-8 E(0) A\left(t_{0}\right)\right\}$ y $b:=8 \gamma^{2} E(0)$. En el caso (i), $t_{0}:=\operatorname{máx}\left\{\frac{A^{\prime}(0)}{4(1+2 \gamma) E(0)}, 0\right\}$ y $t_{0}:=0$ en los casos (ii) y (iii). 
Demostración. De (4.11) y (4.14), resulta

$$
J^{\prime \prime}(t) \leq 4 \gamma(2 \gamma+1) E(0)[A(t)]^{-(\gamma+1)}, \forall t \in\left[t_{0}, T_{M}[\right.
$$

Por (4.8) y resultado del Lema 4.4,

$$
\left.J^{\prime}(t)<0, \forall t \in\right] t_{0}, T_{M}[
$$

Multiplicando (41) por $J^{\prime}(t)$ y luego integrando de $t_{0}$ a $t$, se obtiene

$$
\left[J^{\prime}(t)\right]^{2} \geq a+b[J(t)]^{2+\frac{1}{\gamma}}, \forall t \in\left[t_{0}, T_{M}[.\right.
$$

donde

$$
\begin{aligned}
a & :=\left[J^{\prime}\left(t_{0}\right)\right]^{2}-8 \gamma^{2} E(0)\left[J\left(t_{0}\right)\right]^{2+\frac{1}{\gamma}} \\
& =\gamma^{2}\left[A\left(t_{0}\right)\right]^{-2(\gamma+1)}\left\{\left[A^{\prime}\left(t_{0}\right)\right]^{2}-8 E(0) A\left(t_{0}\right)\right\}
\end{aligned}
$$

y

$$
b:=8 \gamma^{2} E(0) .
$$

Observemos que $a>0$ si y sólo si $E(0)<\frac{\left[A^{\prime}\left(t_{0}\right)\right]^{2}}{8 A\left(t_{0}\right)}$.

En el caso que $E(0)<0$, por (4.11) y (4.14), se obtiene fácilmente la singularidad y la estimativa del tiempo finito $T_{M} \leq t_{0}-\frac{J\left(t_{0}\right)}{J^{\prime}\left(t_{0}\right)}$. Para los demás casos, por (4.17) y Lema 2.2, se obtiene los resultados del Teorema. Esto concluye la demostración.

\section{REFERENCIAS BIBLIOGRÁFICAS}

[1] CARRILLO DÍAZ, L.E., Blow-up em tempo finito para um sistema de equações não lineares, Atas do $30^{\circ}$ SBA, 299 - 308 (1989).

[2] JÖRGENS, K., Nonlinear wave equations, University of Colorado, Department of Mathematics (1970).

[3] LI, M.R. and TSAI, L.Y., On a system of nonlinear wave equations, Taiwanese Journal of Mathematics, Vol. 7, $\mathrm{N}^{\circ}$ 4, 557 - 573 (2003).

[4] MAKHANKOV, V. G., Dynamics of classical solutions in integrable systems, Physics Reports (Section C of Physics Letters) 35, 1 - 128 (1978). 
[5] MEDEIROS, L.A. and MILLA MIRANDA, M., Weak solutions for a system of nonlinear Klein - Gordon equations, Annali di Matematica Pura ed Applicata (IV), Vol. CXLXI, 173 - 183 (1987).

[6] MEDEIROS, L.A. and PERLA MENZALA, G., On a mixed problem for a class of nonlinear Klein - Gordon equations, Atas do $21^{\circ} \mathrm{SBA}(1985)$.

[7] MILLA MIRANDA, M. and MEDEIROS, L. A., On the existence of global solutions of a coupled nonlinear Klein - Gordon equations, Funkcialaj Ekvacioj, Vol. 30, No 147 - 161 (1987).

[8] NISHIHARA, K., On a global solution of some quasilinear hyperbolic equation, Tokio J. Math., Vol. 7, $\mathrm{N}^{\circ} 2,437$ - 459 (1984).

[9] QUISPE MÉNDEZ, T., Singularidad en tiempo finito para un sistema de Kirchhoff, Tesis de Maestría, UNMSM (1998).

[10] SEAL, I., Nonlinear partial differential equations in quantum fields theory, Proc. Symp. Appl. Math. A.M.S., 17, 210 - 226 (1965). 